\title{
Avaliação da microdureza Knoop e tração diametral de resinas compostas de uso direto
}

\section{Evaluating Knoop microhardness and diametral tensile strength of composite resins of direct use}

\author{
Vanessa Fontenele Marques* \\ Élen Carla de Freitas Araújo* \\ Ticiana Pessoa Tabosa e Silva** \\ Maria Cecília Caldas Giorgi ${ }^{* * *}$ \\ Denise Sá Maia Casselli****
}

\section{Resumo}

Objetivo: as propriedades mecânicas dos compósitos odontológicos podem fornecer importantes informações sobre o seu provável desempenho clínico. Nessa perspectiva, este artigo tem por objetivo avaliar a microdureza e a resistência à tração diametral de resinas compostas de uso direto. Materiais e método: foram avaliadas quatro resinas compostas: Filtek Z-250 (3M ESPE, Sumaré, São Paulo, Brasil), Filtek Z-350 XT® (3M ESPE, Sumaré, São Paulo, Brasil), Filtek P-90 (3M ESPE, Sumaré, São Paulo, Brasil) e Opallis ${ }^{\circledast}(F G M$, Joinville, Santa Catarina, Brasil). Foram confeccionados 40 espécimes cilíndricos $(n=10)$ de $4 \mathrm{~mm}$ de diâmetro por $2 \mathrm{~mm}$ de altura. Após a confecção, as amostras foram armazenadas em saliva artificial por $24 \mathrm{~h}$ e, em seguida, foram polidas e submetidas aos ensaios mecânicos. Para o ensaio de dureza, um endentador Knoop foi utilizado com carga de $50 \mathrm{~g}$ por $5 \mathrm{~s}$ nas superfícies de topo e base. Após a mensuração da dureza, as amostras foram submetidas ao teste de tração diametral. Os dados foram analisados por ANOVA e teste de Tukey $(\alpha=0,05)$. Resultados: os resultados obtidos foram: Os compósitos $Z-250^{\circledR}$ e Z-350 X $X T^{\circledast}$ apresentaram os maiores valores de dureza sem diferença estatística entre eles. Não houve diferença entre $P-90^{\odot}$ e Opallis ${ }^{\circledast}$. O ensaio de tração diametral $Z-250^{\odot}$ registrou maiores valores que $P-90^{\odot} \mathrm{e}$ Opallis ${ }^{\circledR}$. Conclusão: com base nesses dados, conclui-se que os compósitos Z-250 $0^{\circledR}$ e Z-350 X $T^{\circledR}$ apresentaram meIhores propriedades mecânicas do que o $P-90^{\circledR}$ e Opallis ${ }^{\circledR}$.

Palavras-chave: Resinas compostas. Dureza. Resistência à tração.

\section{Introdução}

O sucesso das restaurações de resina composta está associado, dentre outros fatores, à escolha adequada do material restaurador ${ }^{1-3}$. A resina escolhida deve apresentar opções de cores, facilidade de manipulação e de polimento, e adequadas propriedades mecânicas. Esse último fator é dependente do tipo de monômero resinoso utilizado ${ }^{4,5}$, da quantidade, do tipo de carga ${ }^{6,7}$, e do processo de polimerização ${ }^{8,9}$ que deve proporcionar alto grau de conversão ${ }^{10,11}$, permitindo que a estrutura polimérica formada apresente uma adequada quantidade de ligações cruzadas $^{12,13}$.

A partir do desenvolvimento do monômero resinoso Bis-GMA (Bis-Fenol A di-Glicidil Metacrilato) por Bowen na década de 1960, foi possível a incorporação de carga aos compósitos odontológicos. Com isso houve grande avanço no desenvolvimento desses materiais e crescente aumento na sua utilização ${ }^{14}$. Diversos avanços foram realizados desde então, tanto na alteração dos monômeros como no desenvolvimento de cargas cada vez menores ${ }^{14-15}$. Das cargas de quartzo de grande tamanho utilizadas nos primeiros compósitos comerciais, evoluiu-se para cargas de tamanho nanométrico em resinas compostas atuais, melhorando a distribuição e a

*** Doutora em Clínica Odontológica pela Faculdade de Odontologia de Piracicaba UNICAMP. Piracicaba, São Paulo, Brasil.

*** Professora adjunta do curso de Odontologia da Universidade Federal do Ceará (UFC) - campus Sobral, Departamento de Dentística. Sobral, Ceará, Brasil. 
quantidade de porção inorgânica ${ }^{16,17}$. Essa evolução busca aumentar as propriedades físicas e mecânicas dos compósitos.

Entretanto, apesar de toda evolução, a resina composta ainda apresenta a contração de polimerização como sua principal limitação clínica, podendo levar ao surgimento de desadaptação marginal ${ }^{18}$, trincas $^{19}$ e sensibilidade pós-operatória nas restaurações ${ }^{1}$. Buscando contornar esses problemas clínicos, novos monômeros resinosos de baixa contração tem sido desenvolvidos ${ }^{1,20,21}$. Dentre esses, 0 silorano, conseguido a partir da união de siloxanos e oxiranos, tem sido utilizado em uma resina comercial da 3M ESPE, denominada no Brasil de Filtek P-90 ${ }^{\circledR}$. Segundo o fabricante, esse material apresen- ta contração volumétrica menor do que $1 \%$ após a sua polimerização. Assim, o objetivo deste estudo foi o de avaliar a dureza Knoop e a resistência à tração diametral de resinas compostas, disponíveis comercialmente no Brasil, com diferentes conteúdos monoméricos e de carga. A hipótese testada é a de que não há diferença entre os valores de dureza e de tração diametral desses materiais.

\section{Materiais e método}

Para realização desta investigação, foram selecionadas quatro resinas compostas de uso direto disponíveis no mercado odontológico (Tab. 1).

Tabela 1 -Materiais utilizados neste estudo e sua composição

\begin{tabular}{|c|c|c|c|}
\hline Resina Composta & Fabricante & Composição ${ }^{1}$ & $\%$ de carga ${ }^{2}$ \\
\hline Filtek Z-250 ${ }^{\circledast}$ & $\begin{array}{l}\text { 3M ESPE } \\
\text { St. Paul, MN, EUA }\end{array}$ & $\begin{array}{l}\text { Bis-GMA, UDMA, Bis-EMA, Sílica /Zircônia }(0,01 \text { a 3,5 } \mu \mathrm{m} \text {, } \\
\text { média 0,6 } \mu \mathrm{m}) \text {. }\end{array}$ & $66 \%$ \\
\hline Filtek Z-350 XT ${ }^{\circledR}$ & $\begin{array}{l}\text { 3M ESPE } \\
\text { St. Paul, MN, EUA }\end{array}$ & $\begin{array}{l}\text { Bis - GMA, UDMA, TEGDMA, Bis-EMA, partículas de Sílica (20 } \\
\text { nm) e Zircônia (4 a } 11 \mathrm{~nm}) \text {. }\end{array}$ & $78,5 \%$ \\
\hline Filtek P-90 ${ }^{\circledast}$ & $\begin{array}{l}\text { 3M ESPE } \\
\text { St. Paul, MN, EUA }\end{array}$ & Silorano, Partículas de quartzo e Fluoreto de ítreo $(0,47 \mu \mathrm{m})$. & $76 \%$ \\
\hline Opallis ${ }^{\circledR}$ & $\begin{array}{l}\text { FGM } \\
\text { Joinvile, SC, Brasil }\end{array}$ & $\begin{array}{l}\text { Bis-GMA, UDMA, Bis-EMA, TEGDMA, Partículas de bário- } \\
\text { alumino silicato e sílica }(0,04 \text { a } 3,0 \mu \mathrm{m} \text {, média } 0,5 \mu \mathrm{m})\end{array}$ & $78,5 \%$ \\
\hline
\end{tabular}

1- Informações dos perfis técnicos disponibilizados pelos fabricantes. 2 - Carga em peso/peso. Bis-GMA - Bis-Fenol A di-Glicidil Metacrilato, Bis-EMA - Bis-Fenol A di-Glicidil Metacrilato etoxilado, TEGDMA - Trietileno glicol dimetacrilato, UDMA - Uretano dimetacrilato.

Para a confecção das amostras, cada resina composta foi inserida em uma matriz de teflon em incrementos menores que $1 \mathrm{~mm}$ de espessura. Essa matriz apresentava $2 \mathrm{~mm}$ de profundidade por $4 \mathrm{~mm}$ de diâmetro. Cada incremento foi fotoativado por 40 segundos, com a ponta do aparelho fotopolimerizador (Degulux Soft Start ${ }^{\circledR}$, Degussa Hills, Hanau, Alemanha) tocando a superfície superior da matriz. No último incremento, uma tira de poliéster foi interposta entre a resina composta e a ponta do aparelho fotopolimerizador. A potência do aparelho $(\cong 700 \mathrm{mw} /$ $\mathrm{cm}^{2}$ ) foi mensurada durante todo o experimento. Dez amostras foram confeccionadas para cada resina composta.

Após a polimerização do último incremento, a amostra foi removida da matriz e armazenada em saliva artificial, ao abrigo da luz, por 24 horas. Após, as superfícies superior e inferior de cada amostra foram polidas com disco de óxido de alumínio (Sof-lex ${ }^{\circledR}, 3$ M ESPE, St. Paul, MN, EUA), utilizados em ordem decrescente de granulação. Após a utilização do disco de menor granulação, as pastas de polimento Diamond ${ }^{\circledR}$ AC I e ACII (FGM, Joinville, SC, Brasil) foram utilizadas, nessa ordem, com auxílio de um disco de feltro.
O ensaio de dureza foi realizado utilizando um microdurômetro (FM 1E Future Tech/Tóquio, Japão) e uma ponta Knoop. A medida de maior diagonal da endentação foi anotada e convertida em valor de dureza Knoop (KHN) pela seguinte fórmula: $\mathrm{KHN}=14.229 \times \mathrm{P} / \mathrm{I}^{2}$, em que $\mathrm{P}=$ Carga aplicada (Carga 50g / 5s); I = Comprimento da maior diagonal da penetração $(\mu \mathrm{m})$. Dez leituras foram realizadas em cada superfície (topo e base), obtendo-se a média dos valores usados na análise estatística em cada superfície.

Os testes de tração diametral foram realizados em uma Máquina Universal de Ensaios (Instron Corporation, Canton, MA, EUA) a uma velocidade de $0,5 \mathrm{~mm} / \mathrm{min}$. As amostras foram posicionados verticalmente na máquina, estando a superfície lateral em contato com a base do dispositivo, e submetidos a uma força de compressão até a fratura. Em segui$\mathrm{da}$, os dados obtidos foram aplicados na fórmula: $\mathrm{R}$ $=2 \mathrm{~L} / \pi \mathrm{Dh}$, em que $\mathrm{R}=$ Valor da tração diametral; $\mathrm{L}=$ Força aplicada $; \mathrm{D}=$ Diâmetro da amostra $; \mathrm{h}=$ altura da amostra. Os dados de altura e de diâmetro foram mensurados com um paquímetro digital. Os dados de dureza e de resistência à tração diametral foram, individualmente, submetidos à ANOVA e ao teste de Tukey $(\alpha=0,05)$. 


\section{Resultados}

No teste de dureza, ANOVA mostrou efeito significante de tratamento apenas para o fator "resina composta" ( $p<0,001)$, enquanto não houve efeito para superfície $(p=0,062)$ e para a interação entre os fatores $(p=0,446)$. Os resultados do teste de Tukey estão descritos na Tabela 2.

Tabela 2 - Média (DP) de valores de dureza em KHN

\begin{tabular}{|c|c|c|c|}
\hline \multirow{2}{*}{ Resina composta } & \multicolumn{2}{|c|}{ Superfície } & \multirow{2}{*}{$\begin{array}{c}\text { Média } \\
\text { agrupada }\end{array}$} \\
\hline & Topo & Base & \\
\hline Filtek Z-250 ${ }^{\circledR}$ & $63,0(11,0)$ & $65,1(10,4)$ & $64,2(10,5) \mathrm{A}$ \\
\hline Filtek Z-350 XT ${ }^{\circledR}$ & $61,4(13,3)$ & $68,8(12,7)$ & $65,1(13,2) \mathrm{A}$ \\
\hline Filtek P-90 ${ }^{\circledR}$ & $35,6(4,0)$ & $34,9(6,1)$ & $35,2(5,0) \mathrm{B}$ \\
\hline Opallis ${ }^{\circledR}$ & $30,8(6,9)$ & $38,5(9,0)$ & $34,6(8,8) \mathrm{B}$ \\
\hline
\end{tabular}

Para a média agrupada, letras distintas indicam diferença estatística $(P<0,05)$.

Os compósitos Filtek Z-250 ${ }^{\circledR}$ e Filtek Z-350 XT ${ }^{\circledast}$ apresentaram os maiores valores de dureza, sem diferença estatística entre si. Os compósitos Filtek $\mathrm{P}-90^{\circledR}$ e Opallis ${ }^{\circledR}$ apresentaram menores valores de dureza, não apresentando diferença entre si.

Para o ensaio de resistência à tração diametral, ANOVA mostrou efeito significativo de tratamento $(p<0,001)$. Os resultados do teste de Tukey estão descritos na Tabela 3 .

Tabela 3 - Resultados de resistência a tração diametral em MPa

\begin{tabular}{l|c}
\hline \multicolumn{1}{c|}{ Resina composta } & Media (DP) \\
\hline Filtek Z-250 & $155,2(24,7) \mathrm{A}$ \\
\hline Filtek Z-350 XT & \\
\hline Filtek P-90 & \\
\hline Opallis $^{\circledR}$ & $127,7(21,7) \mathrm{AB}$ \\
\hline Letras distintas indicam diferença estatística $(p<0,05) .^{1}$ & $110,9(17,4) \mathrm{B}$ \\
\hline
\end{tabular}

O compósito Filtek Z-250 apresentou maiores valores de resistência à tração diametral que Filtek P-90 ${ }^{\circledR}$ e Opallis $^{\circledR}$. Enquanto que a Filtek Z-350 XT ${ }^{\circledR}$ apresentou valores que não diferem estatisticamente da Filtek Z-250 ${ }^{\circledR}$ com melhor resultado, nem das demais com resultado inferior.

\section{Discussão}

A escolha da resina composta para procedimentos restauradores diretos deve se basear em importantes parâmetros, que podem resultar no aumento da longevidade da restauração $0^{1-3}$. A avaliação das propriedades mecânicas pode ajudar a predizer se a resina composta escolhida irá suportar as cargas mastigatórias sem fraturar ou desgastar. Dentre os ensaios mecânicos comumente utilizados, o ensaio de dureza pode predizer se o material restaurador irá resistir ao desgaste, considerando que materiais com baixa dureza são mais susceptíveis ao desgaste ${ }^{12,22}$. Já o teste de tração diametral mostra a capacidade da resina composta em suportar cargas de compressão, como, por exemplo, as cargas oclusais que geram tensões de tração, sem fraturar ${ }^{8,9}$. Este estudo mostrou diferença, tanto nos valores de dureza como nos de resistência à tração diametral, entre compósito de diferentes composições monoméricas e de carga. Assim, a hipótese testada foi rejeitada.

Os valores de dureza de um compósito estão relacionados, dentre outros fatores, ao grau de conversão do material, também utilizados como uma forma indireta de predizer a conversão de monômeros em polímeros ${ }^{8,9,11}$. Um maior grau de conversão de um material resinoso estaria associado a um maior valor de dureza. Apesar dessa relação ser mais precisa em materiais de mesma composição monomérica e de carga, parece pertinente associar os resultados encontrados a diferenças no processo de polimerização das resinas compostas $^{10,12,13}$. Exceção feita à Filtek P-90 ${ }^{\circledR}$, os compósitos avaliados apresentam semelhança de componentes monoméricos, que seriam um dos fatores importantes no processo de polimerização. Entretanto, a exata composição desses materiais, como a concentração de cada monômero, é pouco conhecida em compósitos comerciais, tornando qualquer discussão de resultados baseada nos componentes monoméricos meramente especulativa. Da mesma forma, a informação disponibilizada pelos fabricantes sobre o tipo e a concentração de iniciadores de reação de polimerização desses materiais é escassa.

Em relação à resistência à tração diametral, tem sido demonstrado que o aumento na quantidade de carga inorgânica melhora as propriedades mecânicas das resinas compostas ${ }^{6,23}$. Entretanto, essa relação direta entre quantidade de carga e resistência mecânica apenas ocorre até o limite de $55 \%$ (peso/peso), a partir do qual novos incrementos de carga inorgânica não resultam em aumento significativo de resistência mecânica ${ }^{6}$. Todas as resinas compostas utilizadas nesse estudo apresentam quantidade de carga superior a $55 \%$ em peso. Assim, da mesma forma que para a dureza, as diferenças nos valores de resistência à tração diametral estão, também, provavelmente associadass ao grau de conversão dos compósitos. Isso pode ser corroborado pela semelhança na ordenação das resinas compostas para os valores de dureza e resistência à tração diametral. Baseado nos resultados deste trabalho, os compósitos Filtek Z-250 ${ }^{\circledR}$ e Filtek Z-350 XT ${ }^{\circledR}$ apresentam melhores propriedades mecânicas que o as resinas compostas Filtek P-90 ${ }^{\circledR}$ e Opallis ${ }^{\circledR}$, quando utilizadas sob o mesmo protocolo de fotoativação. 


\section{Conclusão}

Os compósitos Filtek Z-250 ${ }^{\circledR}$ e Filtek Z-350 $\mathrm{XT}^{\circledast}$ apresentaram maiores valores de dureza que a Filtek P-90 $0^{\circledast}$ e Opallis ${ }^{\circledast}$. A resina composta Filtek $\mathrm{Z}-250^{\circledR}$ apresentou a maior resistência à tração diametral, com diferença significativa para os compósitos Filtek P-90 ${ }^{\circledast}$ e Opallis ${ }^{\circledR}$.

\section{Abstract}

The mechanical properties of dental composites can provide important information on their probable clinical performance. Objective: in this perspective, this article aims to evaluate both microhardness and the diametral tensile strength of composite resins of direct use. Materials and method: four composite resins were evaluated: Filtek Z-250 ${ }^{\circledR}$ (3M ESPE, Sumaré, São Paulo, Brazil), Filtek Z-350 XT® (3M ESPE, Sumaré, São Paulo, Brazil), Filtek P-90 (3M ESPE, Sumaré, São Paulo, Brazil) and Opallis ${ }^{\circledR}$ (FGM, Joinville, Santa Catarina, Brazil). Forty cylindrical specimens were prepared $(n=10)$ whose measures were $4 \mathrm{~mm}$ in diameter and $2 \mathrm{~mm}$ in height. After preparation, the samples were stored in artificial saliva for 24 hours and then polished and subjected to mechanical tests. A Knoop indenter was used for the hardness test with a load of $50 \mathrm{~g}$ for 5 seconds at the top and bottom surfaces. After measuring the hardness, the samples were subjected to diametral tensile strength test. The resulting data were analyzed by ANOVA and the Tukey test $(\alpha=0.05)$. Results: the results obtained were as follows: The Z-250 ${ }^{\circledR}$ and Z-350 XT composites showed higher hardness values without statistical difference between them. There was no difference between $P-90^{\circledR}$ and Opallis ${ }^{\circledR}$. In diametrical tensile strength test, $Z-250^{\circledast}$ presented higher values than $P-90^{\circledR}$ and Opallis ${ }^{\circledR}$. Conclusion: based on these results, we conclude that Z-250 and Z-350 X $T^{\circledR}$ composites showed better mechanical properties than $P-90^{\circledR}$ and Opallis ${ }^{\circledR}$.

Keywords: Composite resins. Hardness. Tensile strength.

\section{Referências}

1. Baracco B, Perdigão J, Cabrera E, Giráldez I, Ceballos L. Clinical evaluation of a low-shrinkage composite in posterior restorations: one-year results. Oper Dent 2012; 37(2):117-29.

2. Demarco FF, Corrêa MB, Cenci MS, Moraes RR, Opdam NJ. Longevity of posterior composite restorations: not only a matter of materials. Dent Mater 2012; 28(1):87-101.

3. Gonçalves FS, Castro CD, Bueno AC, Freitas AB, Moreira AN, Magalhaes CS. The short-term clinical performance of a silorane-based resin composite in the proximal contacts of class II restorations. J Contemp Dent Pract 2012; 13(3):251-6.

4. Gonçalves F, Kawano Y, Pfeifer C, Stansbury JW, Braga RR. Influence of BisGMA, TEGDMA, and BisEMA contents on viscosity, conversion, and flexural strength of experimental resin and composites. Eur J Oral Sci 2009; 117(4):442-6.

5. Kilambi H, Cramer NB, Schneidewind LH, Shah P, Stansbury JW, Bowman CN. Evaluation of highly reactive mono-(meth)acrylates as reactive diluents for BisGMA-based dental composites. Dent Mater [periódico on-line]. 2009 Jan [acesso em 19 abr. 2013]; 25(1):33-8.

6. Kim KH, Ong JL, Okuno O. The effect of filler loading and morphology on the mechanical properties of contemporary composites. J Prosthet Dent 2002; 87(6):642-9.

7. Melander J, Dunn WP, Link MP, Wang Y, Xu C, Walker MP. Comparison of flexural properties and surface roughness of nanohybrid and microhybrid dental composites. Gen Dent 2011; 59(5):342-7.

8. Aguiar FH, Braceiro AT, Ambrosano GM, Lovadino JR. Hardness and diametral tensile strength of a hybrid composite resin polymerized with different modes and immersed in ethanol or distilled water media. Dent Mater 2005; 21(12):1098-103.

9. Casselli DS, Worschech CC, Paulillo LA, Dias CT. Diametral tensile strength of composite resins submitted to different activation techniques. Braz Oral Res [periódico on-line] 2006 Jul-Sep [acesso em 19 abr. 2013]; 20(3):214-8.

10. Albino LG, Rodrigues JA, Kawano Y, Cassoni A. Knoop microhardness and FT-Raman evaluation of composite resins: influence of opacity and photoactivation source. Braz Oral Res [periódico on-line]. 2011 May-Jun [acesso em 19 abr. 2013]; 25(3):267-73.

11. Kusgoz A, Ülker M, Yesilyurt C, Yoldas OH, Ozil M, Tanriver M. Silorane-based composite: depth of cure, surface hardness, degree of conversion, and cervical microleakage in Class II cavities. J Esthet Restor Dent 2011; 23(5):324-35.

12. Benetti AR, Peutzfeldt A, Asmussen E, Pallesen U, Franco EB. Influence of curing rate on softening in ethanol, degree of conversion, and wear of resin composite. Am J Dent 2011; 24(2):115-8.

13. Park J, Eslick J, Ye Q, Misra A, Spencer P. The influence of chemical structure on the properties in methacrylate-based dentin adhesives. Dent Mater [periódico on-line]. 2011 Nov [acesso em 19 abr. 2013]; 27(11):1086-93.

14. Ilie N, Hickel R. Resin composite restorative materials. Aust Dent J [periódico on-line]. 2011 Jun [acesso em 19 abr. 2013]; 56 Suppl 1:59-66.

15. Ferracane JL. Buonocore Memorial Lecture. Placing dental composites - a stressful experience. Oper Dent [periódico on-line]. 2008 May-Jun [acesso em 19 abr. 2013]; 33(3):247-57.

16. Garoushi S, Lassila LV, Vallittu PK. Influence of nanometer scale particulate fillers on some properties of microfilled composite resin. J Mater Sci Mater Med 2011; $22(7): 1645-51$.

17. Takahashi H, Finger WJ, Endo T, Kanehira M, Koottathape $\mathrm{N}$, Komatsu M, et al. Comparative evaluation of mechanical characteristics of nanofiller containing resin composites. Am J Dent 2011;24(5):264-70.

18. Maia-Casseli DS, Faria-e-Silva AL, Cavalcanti AN, Romani EA, Martins LR. Effect of light-curing unit and adhesive system on marginal adaptation of class $\mathrm{v}$ composite restorations. Acta Odontol Latinoam 2012; 25(1):68-73.

19. Jafarpour S, El-Badrawy W, Jazi HS, McComb D. Effect of composite insertion technique on cuspal deflection using an in vitro simulation model. Oper Dent 2012; 37(3):299-305.

20. Tantbirojn D, Pfeifer CS, Braga RR, Versluis A. Do low-shrink composites reduce polymerization shrinkage effects? J Dent Res. 2011; 90(5):596-601. 
21. Yamamoto T, Kubota Y, Momoi Y, Ferracane JL. Polymerization stresses in low-shrinkage dental resin composites measured by crack analysis. Dent Mater 2012; 28(9):e143-9.

22. Suwannaroop P, Chaijareenont P, Koottathape N, Takahashi $\mathrm{H}$, Arksornnukit M. In vitro wear resistance, hardness and elastic modulus of artificial denture teeth. Dent Mater J [periódico on-line]. 2011[acesso em 19 abr. 2013]; 30(4):461-8.

23. Rastelli AN, Jocomassi DP, Faloni AP, Queiroz TP, Rojas SS, Bernardi MI, et al. The filler contente of the dental composite resins and their influence on diferente properties. Microsc Res Tech 2012; 75(6):756-8.

\section{Endereço para correspondência:}

Denise Sá Maia Casselli

Rua Coronel Estanislau Frota, S/N - Cento

62.010-560 Sobral-CE

Fone: (88) 36132603

E-mail:dsmaia@yahoo.com

Recebido: 17/03/2014. Aceito: 10/08/2014. 